\title{
Evaluation of a TDMA-based energy efficient MAC protocol for multiple capsule networks
}

\author{
Lin Lin ${ }^{1,2}$, Kai-Juan Wong ${ }^{2 *}$, Arun Kumar², Zongqing Lu², Su-Lim Tan² and Soo Jay Phee
}

\begin{abstract}
Wireless capsule endoscopy is a new kind of medical device, which monitors the gastrointestinal tract of the human body. It can be envisaged that in the future more than one capsule could be ingested by the patient and they operate collaboratively in the gastrointestinal (Gl) tract to perform certain diagnostic and therapeutic tasks. These mobile capsules and the coordinator node, which is attached to the abdomen of the patient, form a wireless network. The capsule devices are typically powered by batteries, therefore, energy efficient medium access control (MAC) protocols for multiple capsule networks are necessary. This article proposes a novel energy efficient MAC protocol for multiple capsule networks based on time division multiple access (TDMA). An asymmetric up/ down link network architecture is introduced. A novel TDMA slot assignment scheme is proposed and simulation results using Qualnet show that the proposed MAC protocol achieves lower energy consumption than B-MAC and star topology TDMA.
\end{abstract}

Keywords: multiple capsule networks, MAC protocol, TDMA, asymmetric topology

\section{Introduction}

Wireless capsule endoscopy (WCE) is a new kind of medical device, which is ingested by the patient for the purpose of inspecting the gastrointestinal (GI) tract. Currently, the commercial WCE is mainly composed of a camera, a transceiver, and two button batteries [1]. The camera captures images of the GI tract and sends them to an external data recorder wirelessly. It is envisaged that, in the future, WCE will be made more versatile by providing many advanced functionalities such as active locomotion, tissue sampling, and drug delivery. It could also be imagined that several capsules co-operate in the GI tract to monitor the vital body signs or to perform a common task [2]. These capsules and the data recorder (coordinator node), which is externally attached to the abdomen, form a wireless network that can be viewed as a subset of body sensor networks (BSN). Figure 1 shows an example of the WCE and multiple capsule networks.

The current capsule devices are powered by two $1.5 \mathrm{~V}$, $80 \mathrm{mAh}$ button batteries. It can last $8 \mathrm{~h}$ to perform only the basic function of capturing and transmitting images

\footnotetext{
*Correspondence: askjwong@ntu.edu.sg

${ }^{2}$ School of Computer Engineering, Nanyang Technological University, Singapore

Full list of author information is available at the end of the article
}

with a frame rate of 2 frames/s [3]. Efficient utilization of the limited energy is an issue. It is a well known fact that the radio transceiver consumes a large part of the energy budget of wireless sensor devices [4] and a good medium access control (MAC) protocol can efficiently reduce the energy consumed by the transceiver. Many energy efficient MAC protocols have been proposed for wireless sensor networks (WSN) and BSN [5-9]. However, multiple capsule networks have some unique properties such as mobility, the size of the operating area, scalability, safety, reliability, etc., so specific energy efficient MAC protocols for multiple capsule networks are necessary.

This article proposes a novel energy efficient MAC protocol for multiple capsule networks based on time division multiple access (TDMA). The multiple capsule networks operate only within the body area, thus, the coordinator radio transmission can cover the entire network area and control all the capsule devices directly. Based on this, the article proposes a novel up/down link asymmetric network architecture. For the downlink data transmissions, the coordinator node sends data directly to each sensor device, while for the uplink communications, data are sent via multi-hop mode to the coordinator node. In this way, the power consumption can be

\section{SpringerOpen ${ }^{\odot}$}

(C) 2011 Lin et al; licensee Springer. This is an Open Access article distributed under the terms of the Creative Commons Attribution License (http://creativecommons.org/licenses/by/2.0), which permits unrestricted use, distribution, and reproduction in any medium, provided the original work is properly cited. 

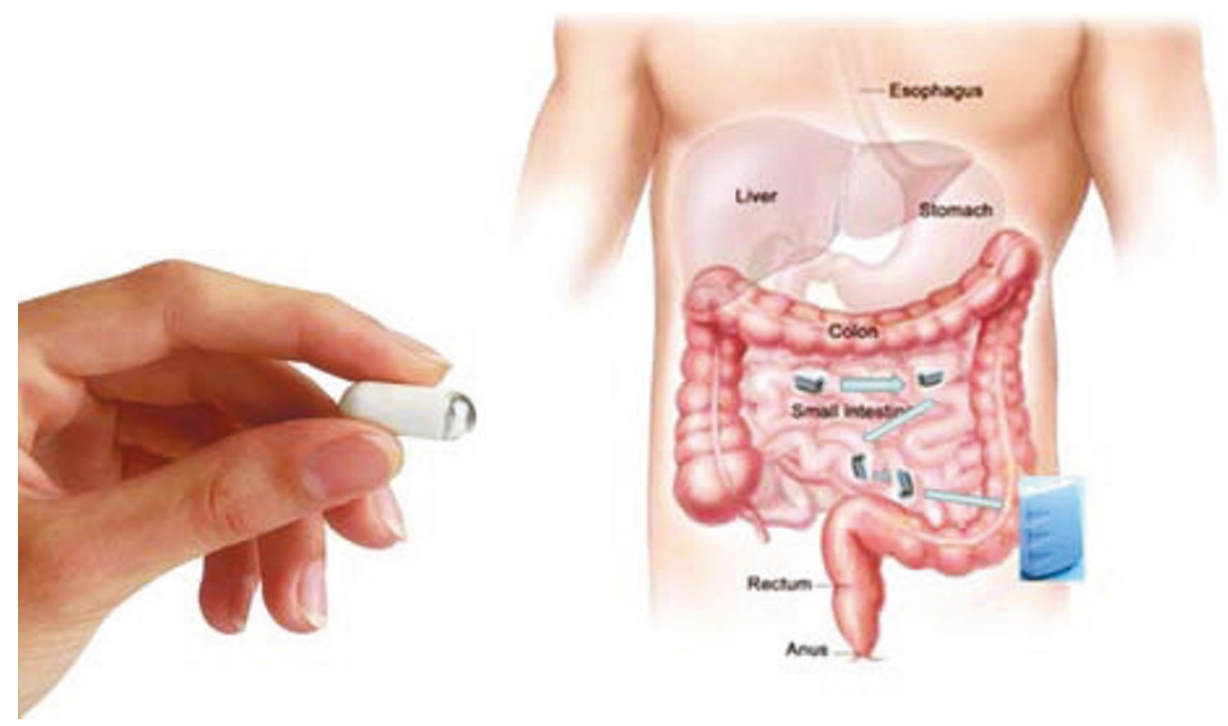

Figure 1 Wireless capsule endoscopy and multiple capsule networks.

reduced. A TDMA scheduling scheme is proposed. Each capsule periodically collects the neighboring information and sends this information to the coordinator node. The coordinator node is the processing center of the whole network. It calculates the best route and time slot schedules, and then sends the schedules back to the capsules. Capsules only wake up in their own slots and go into sleep status for the rest. Adaptive power control is used in this proposed protocol to further reduce the energy consumption. Simulation results obtained using the Qualnet simulator showed that the proposed MAC protocol achieves better performance than B-MAC and star topology TDMA in terms of energy consumption.

The rest of the article is organized as follows: Section 2 introduces the related works. Section 3 describes details of the protocol design with the evaluation of its performance presented in Section 4. Finally, Section 5 concludes the article.

\section{Related works}

In WSN, energy wastage is mainly due to collisions, idle listening, overhearing, and overhead. Many energy efficient MAC protocols have been proposed in prior works. B-MAC [5], S-MAC [6], T-MAC [10] are examples of typical contention-based MAC protocols. They offer the advantages of simplicity, small overhead, small latency, etc. TRAMA [11] and LMAC [12] are typical schedule-based MAC protocols. Compared with contention-based MAC protocol, schedule-based MAC protocols can avoid collisions, idle listening, and overhearing very easily. BSN, compared with WSN, has a limited number of nodes that are attached on the human body or implanted into the body. The data are gathered from heterogeneous medical sensors with batteries that are more difficult to replace. Marinkovic et al. [9] proposed an energy efficient low duty cycle MAC protocol. It adopts the TDMA-based strategy and star topology. The sensor nodes go into sleep mode when they do not have data to send or receive. However, the pure star topology used consumes much more energy to transmit sensing data to the coordinator. HyungTae et al. [13] proposed an energy efficient multi-hop MAC protocol. Minimum spanning tree routing is utilized and a dynamic time slot allocation is proposed. The protocol is not well suited to the networks with mobile sensor devices due to the requirement for fixed data transmission power. This article adopts the TDMA schedule-based mode for mobile capsule nodes. An up/down link asymmetric topology and adaptive power control are used. The power consumption of the sensor devices is reduced significantly.

\section{Protocol design}

\subsection{Overview and attributes}

Multiple capsule networks are composed of a coordinator node and several capsules moving along the GI tract. It has several attributes. First, the path-loss of the human body is quite big due to the different electrical properties of the body tissue [14]; therefore, wireless communication through the human body is more challenging than through the air. Therefore, higher transmit power is needed. Secondly, because the capsules are of a small size around $26 \times 11 \mathrm{~mm}$ (length $\times$ diameter), the power and resources for processing on the capsule are very limited. The coordinator node is typically outside the human body without size limitation and the 
batteries are easily replaced, hence the power source for the coordinator node can be considered to be unlimited. Thirdly, many sensors could be integrated into capsules to monitor vital body signs such as temperature, blood pressure, electrocardiography (ECG), images of the GI tract. These sensors may have disparate sampling rate and sample data size. For example, the pressure and temperature sampled data may be smaller in size and lower in sampling rate than the real time image data. Finally, unlike most WSN, multiple capsule networks operate only within the area of the body. This makes the capsule devices easily reachable from the coordinator. Based on these unique attributes, the network architecture, the access and sleep scheme, routing and duty cycle are discussed below.

\subsection{Asymmetric network architecture}

This article proposes a novel asymmetric up/down link topology. It is a mixture of the centralized architecture and the distributed architecture (Figure 2). For downlink data transmissions, because the coordinator node is outside the human body with no strict constrain in size, the radio of the coordinator node could easily cover all the capsule devices. Based on this, a centralized architecture is adopted. The coordinator node is assumed to have unlimited energy and thus, its energy consumption is not considered. The total energy consumed by the capsules to receive data from the coordinator node directly can be calculated as the receiving energy consumption of the capsule device, $E_{\mathrm{r}}$. If the coordinator node sends data by a multi-hop way through node group $K$, then the total energy consumption is equal to $E_{\mathrm{r}_{i}} . E_{\mathrm{r}_{i}}$ and $E_{\mathrm{t}_{i}}$ are the receiving energy consumption and the transmitting energy consumption of node $i(i \in$ $K)$, respectively. Obviously the energy consumption of the downlink multi-hop communication is larger than the energy consumption of direct transmission.

For uplink data transmission, because the capsule devices have very limited power and the batteries are not easily replaced, and the path-loss inside the human body is large, multi-hop communication is considered. In Equation 1, $E_{\text {single }}$ is the energy consumed by the capsule node, which transmits the data directly to the coordinator node. It is equal to the summation of $E_{\mathrm{c}}$

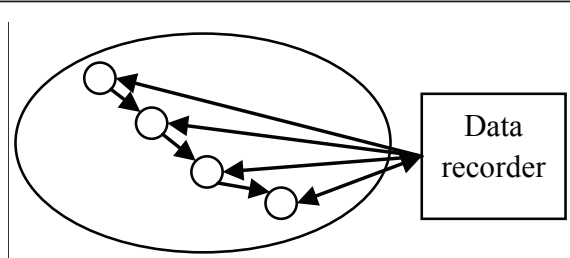

Figure 2 Asymmetric link topology and $E_{\mathrm{t}_{\text {single }}}$. $E_{\mathrm{c}}$ represents the energy consumed by the circuitry (i.e., circuitry power) and $E_{\mathrm{t}_{\text {single }}}$ is the transmit energy. In Equation 2, $E_{i j}$ represents the total energy consumed by node $i$ which transmits data to node $j$. It is equal to the summation of $E_{\mathrm{c}}$ and $E_{\mathrm{t}_{i j}}$. $E_{\mathrm{t}_{i j}}$ is the transmit energy for sensor node $i$ to transmit data to sensor node $j . \sum_{n} E_{i j}$ is the total transmit energy consumption for multi-hop communication. The multi-hop communication can save energy only if $\sum_{n} E_{i j}$ is smaller than $E_{\text {single }}$ (Equations 3 and 4).

$$
\begin{aligned}
& E_{\text {single }}=E_{\mathrm{c}}+E_{\mathrm{t}_{\text {single }}} \\
& E_{i j}=E_{\mathrm{c}}+E_{\mathrm{t}_{i j}} \\
& \sum_{n} E_{i j} \leq E_{\text {single }} \\
& E_{\mathrm{C}}+E_{\mathrm{t}_{\text {single }}} \geq \sum_{n}\left(E_{\mathrm{c}}+E_{\mathrm{t}_{i j}}\right)=n \times E_{\mathrm{C}}+\sum_{n} E_{\mathrm{t}_{i j}}
\end{aligned}
$$

To evaluate the energy consumption for multi-hop communications through the human body, a series of scenarios of different capsules are set up based on a topology of straight line within the range of $30 \mathrm{~cm}$ in Figure 3. The capsules are uniformly distributed. The simulation parameters are shown in Table 1. Figure 4 gives the total energy consumption vs. the number of hops for different circuitry power. Figure 4a shows the total energy consumption at the circuitry power of $10 \mathrm{~mW}$. It can be seen that multi-hop communication consumes less energy than single hop communication. As the circuitry power goes smaller, the multihop communication becomes more meaningful as shown in Figure 4b. According to McGregor et al. [15], at the data rate of $1 \mathrm{Mbps}$, the circuitry power consumption can reach $33 \mu \mathrm{W}$, so four/five hop communication can be used to save energy for the uplink data transmission. As discussed above, the uplink, downlink asymmetric topology gives better performance in terms of the energy consumption.

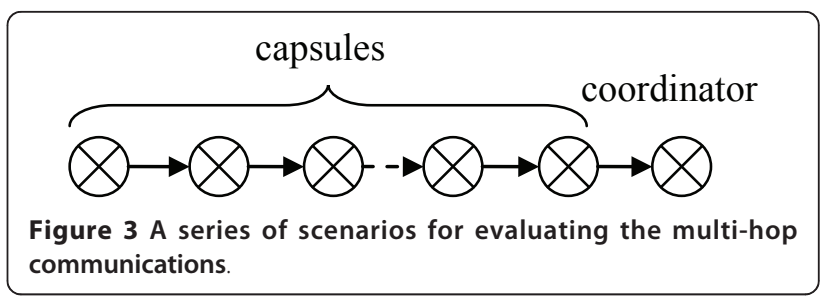


Table 1 Parameters for evaluating the multi-hop communications

\begin{tabular}{llll}
\hline Parameters & Value & Parameters & Value \\
\hline Area & Straight line of $30 \mathrm{~cm}$ & Inefficiency factor $\alpha$ & 6.5 \\
Number of capsules & 1 to 8 & Channel frequency & $405 \mathrm{MHz}$ \\
Pathloss model & Pathloss matrix & Items to send & 1000 \\
Radio type & ABSTRACT & Packet size & 1536 bytes \\
$\begin{array}{l}\text { Transmission power } \\
(\mathrm{dBm})\end{array}$ & Adaptive & CBR packet interval & $0.05 \mathrm{~s}$ \\
$\begin{array}{l}\text { Energy model } \\
\text { Transmission power }\end{array}$ & GENERIC & Simulation time & $30 \mathrm{~s}$ \\
$(\mathrm{dBm})$ & $15.5,-14.5,-24.5,-29.5,-32.5,-34.5,-35.9286$, & Transmit/receive circuitry & $1.5 \mu \mathrm{W}, 10 \mu \mathrm{W}, 30 \mu \mathrm{W}, 100 \mu \mathrm{W}, 10$ \\
\hline
\end{tabular}

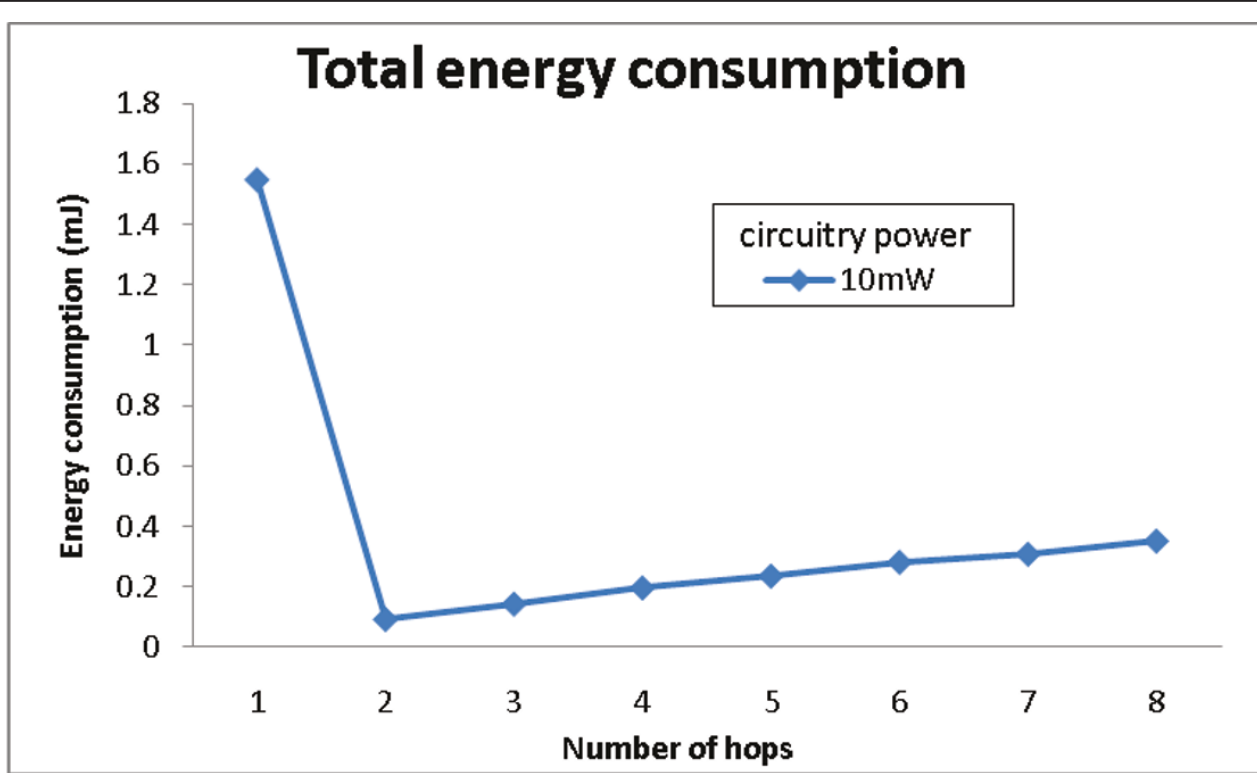

(a)

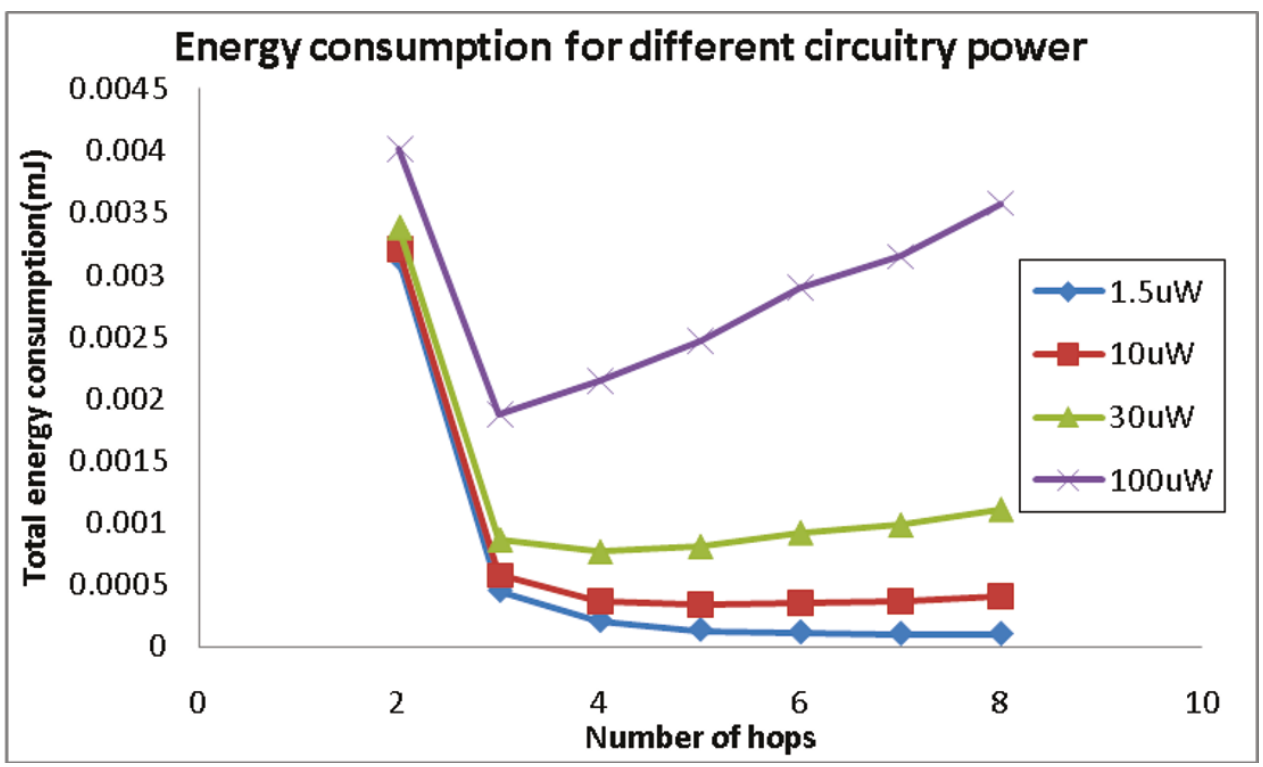

(b)

Figure 4 Total energy consumption for different circuitry power consumption 


\subsection{TDMA Frame format}

In multiple capsule networks, because the wireless coverage of the coordinator node can reach all the capsule devices, network synchronization can be easily achieved. Therefore, a TDMA-based MAC protocol is proposed. The TDMA scheme can avoid collision, idle listening, and overhearing. It can also maximize the bandwidth utilization.

In the proposed MAC protocol, time slots are assigned by the coordinator node. Figure 5 shows the frame format of the TDMA frame. It is composed of a number of control slots and data slots. Control slots include synchronization slot, broadcast slot, power detection slots, neighboring information upload slots, and schedule assignment slots. Synchronization information is broadcasted by the coordinator node in the beginning of the frame. All sensors must keep listening in the first time slot in order to be synchronized with the whole network. In the second time slot, the coordinator broadcasts the control section schedules for all the capsules. It gives the starting slot for power detection, neighboring information uploading, and schedule assignment, respectively. All the capsules receive this broadcast information and use its own identification (ID) as the shift to calculate their own transmit time slot for power detection, neighboring information uploading, and schedule assignment. In the power detection section, each sensor broadcasts its own information including sensor ID and transmit power in its own time slot. During the rest of the time, it listens for the power detection information of other nodes. In this section, the transmit power must be large enough to ensure that all the other capsules can hear and receive this neighboring information. In the upload section, the capsules send the collected information and its time slot request to the coordinator node. They go into sleep mode when other nodes send upload data to the coordinator node in order to save energy. After coordinator node receives all these neighboring information and time slot request, it begins to calculate routing and slot schedule pattern. The time slot assignment is completely flexible. If a capsule has a lot of data to send, then it would be assigned more data slots. If a capsule has no data to send, then it would not be assigned data slots. In the schedule assignment section, the coordinator node sends the schedules to the capsules. The capsules only receive in their own slots and during the rest of the time in this section, they are in the sleep mode. The schedules includes information about the transmit time slot, receive time slot, and the transmit power for the specific capsule in the following data slot section. In the data slot section, the capsule devices follow the received schedules to complete the communications. The whole process repeats in the next TDMA frame. Figure 6 shows an example of TDMA frame for four capsules in the network. The packet structures of the control data are given in Figure 7.

\subsection{Routing calculation and adaptive transmit power control}

All the neighboring information are collected by the capsule devices and sent to the coordinator node with time slot request. The coordinator node calculates the routes, transmit power, and the slot assignment for each capsule. From the neighboring information, the pathloss, PL, of any two sensors can be calculated according to Equation 5 using the transmit power and received signal strength indication (RSSI). $P_{\mathrm{t}}$ is the transmit power from node $i$ to node $j$. RSSI is the receiving power of node $j$. The minimum power consumption, $P_{\mathrm{t}_{i j}}(\mathrm{~dB})$, of any two sensor devices can be calculated according to Equations 6 and 7. The coordinator node generates the matrix of all the possible routes. For each possible route, the total energy consumption is calculated as Equation 8. $\sum_{\text {route }} P_{\mathrm{t}_{i j}}$ is the summation of transmit power in the route. $\sum_{\text {route }} P_{\mathrm{r}_{i j}}$ is the summation of receive power except the coordinator node. The receive power consumption of the coordinator node is excluded because the coordinator node is outside the human body, so it is assumed the coordinator node has unlimited power. The coordinator node calculates the total power consumption of each possible route and finds the smallest one. For this chosen route, the corresponding transmit power can be calculated according to Equation 6. Algorithm 1 shows the route calculation algorithm.

$$
\begin{aligned}
& \mathrm{PL}=P_{\mathrm{t}}-\text { RSSI } \\
& P_{\mathrm{t}_{i j}}(\mathrm{~dB})=\text { Sensitivity }+\mathrm{PL}_{i j}+P_{\text {guard }}(\mathrm{dB})
\end{aligned}
$$

\begin{tabular}{|l|l|l|l|l|l|l|l|l|l|l|l|l|l|l|l|l|l|l|l|}
\hline 1 & 2 & 3 & & $\mathrm{~N}+2$ & $\mathrm{~N}+3$ & & & $2 \mathrm{~N}+2$ & & & $2 \mathrm{~N}+5$ & & $3 \mathrm{~N}+4$ & & & \\
\hline sync & bcst & powload & & & schedule assignment & data slots \\
\hline \multicolumn{8}{|c|}{ control data } \\
\hline
\end{tabular}

Figure 5 TDMA frame format. 


\begin{tabular}{|c|c|c|c|c|c|c|c|c|c|c|c|c|c|c|c|c|c|c|}
\hline & 1 & 2 & 3 & 4 & 5 & 6 & 7 & 8 & 9 & 10 & 11 & 12 & 13 & 14 & 14 & 16 & 51 & 69 \\
\hline 1 & ctx & ctx & $r x$ & $r x$ & $r x$ & $r x$ & $r x$ & $r x$ & $r x$ & $r x$ & & & ctx & ctx & ctx & ctx & & \\
\hline 2 & $r x$ & $r x$ & ctx & $r x$ & $r x$ & $r x$ & ctx & sleep & sleep & sleep & & & $r x$ & sleep & sleep & sleep & & \\
\hline 3 & $r x$ & $r x$ & $\mathrm{rx}$ & ctx & $r x$ & $r x$ & sleep & ctx & sleep & sleep & & & sleep & rx & sleep & sleep & & \\
\hline 4 & $r x$ & $r x$ & $r x$ & $r x$ & $\operatorname{ctx}$ & $r x$ & sleep & sleep & ctx & sleep & & & sleep & sleep & $r x$ & sleep & & \\
\hline \multirow[t]{2}{*}{5} & $r x$ & $r x$ & $r x$ & $r x$ & $r x$ & ctx & sleep & sleep & sleep & ctx & & & sleep & sleep & sleep & $r x$ & & \\
\hline & sync & & \multicolumn{4}{|c|}{ power detection } & \multicolumn{4}{|c|}{ upload } & & & \multicolumn{4}{|c|}{ schedule assignment } & \multicolumn{2}{|c|}{ data slots } \\
\hline
\end{tabular}

Figure 6 Frame format for a four sensor BSN.

$$
\begin{aligned}
& P_{\mathrm{t}_{i j}}=10 \frac{P_{\mathrm{t}_{i j}}(\mathrm{~dB})}{10} \\
& P_{\mathrm{tmp}}=\sum_{\text {route }} P_{\mathrm{t}_{i j}}+\sum_{\text {route }} P_{\mathrm{r}_{i j}}
\end{aligned}
$$

Algorithm 1 Route calculation

SET $P_{\text {comp }}<=1000000, i=0$

WHILE $i<$ number of total possible route do

$P_{\text {tmp }}<=0$

$P_{\mathrm{t}_{i j}}(\mathrm{~dB})=$ Sensitivity $+\mathrm{PL}_{i j}+P_{\text {guard }}(\mathrm{dB})$

$P_{\mathrm{t}_{i j}}=10^{\wedge}\left(P_{\mathrm{t}_{i j}}(\mathrm{~dB}) / 10\right)$

$P_{\mathrm{tmp}}=\sum P_{\mathrm{t}_{i j}}+\sum P_{\mathrm{r}}$ If $P_{\mathrm{tmp}}<P_{\text {comp }}$ Then

$P_{\text {comp }}=P_{\text {tmp }}$

record this route

End if

$i=i+1$

End While

\subsection{Duty cycle analysis}

Duty cycle is a very important concept in energy efficient MAC protocol design. It refers to the percentage of time in active status [9]. It is computed as Equation 9.

$$
\mathrm{DC}=\frac{T_{\text {active }}}{T_{\text {frame }}} \times(1+\text { PER })
$$

where $T_{\text {active }}$ is the time duration of active status within one TDMA frame. $T_{\text {frame }}$ is the TDMA frame duration. PER is the packet error rate.

$$
T_{\text {active }}=T_{\text {data }}+T_{\text {oh }}+\frac{T_{\text {sync }}}{N_{\mathrm{R}}}
$$

where $T_{\text {data }}$ is the time duration for data transmission. $T_{\text {oh }}$ is the time duration for overhead transmission. $T_{\text {sync }}$ is the time duration for synchronization transmission and $N_{\mathrm{R}}$ is TDMA resynchronization rate.

$$
\begin{aligned}
& \text { DC }=\frac{T_{\text {data }}+T_{\text {oh }}+\frac{T_{\text {sync }}}{N_{\mathrm{R}}}}{T_{\text {frame }}} \times(1+\text { PER }) \\
& =\frac{N_{\text {data }}+N_{\text {oh }}+\frac{N_{\text {sync }}}{N_{\mathrm{R}}}}{f_{\mathrm{c}} \times T_{\text {frame }}} \times(1+\text { PER }) \\
& =\frac{T_{\text {frame }} \times f_{\mathrm{s}}+N_{\text {oh }}+\frac{N_{\text {sync }}}{N_{\mathrm{R}}}}{f_{\mathrm{c}} \times T_{\text {frame }}} \times(1+\text { PER })
\end{aligned}
$$

\section{Broadcast packet structure \\ Neighboring information \\ packet structure}

\begin{tabular}{|c|c|c|c|c|}
\hline $\begin{array}{c}\text { number of } \\
\text { sensors }\end{array}$ & $\begin{array}{c}\text { slot for } \\
\text { power } \\
\text { detection }\end{array}$ & $\begin{array}{c}\text { slot for } \\
\text { uploading }\end{array}$ & $\begin{array}{c}\text { slot for } \\
\text { schedule } \\
\text { assignment }\end{array}$ & TB \\
\hline
\end{tabular}

Power detection packet structure

\begin{tabular}{|c|c|c|}
\hline $\begin{array}{c}\text { own } \\
\text { sensor } \\
\text { ID }\end{array}$ & $\begin{array}{c}\text { transmit } \\
\text { power }\end{array}$ & TB \\
\hline
\end{tabular}

\begin{tabular}{|l|c|c|c|c|}
\hline sensor ID & $\begin{array}{c}\text { tx } \\
\text { power }\end{array}$ & RSSI & $\begin{array}{c}\text { slot } \\
\text { request }\end{array}$ & TB \\
\hline
\end{tabular}

\section{Schedule assignment packet structure}

\begin{tabular}{|c|c|c|c|c|c|c|c|}
\hline $\begin{array}{c}\text { source } \\
\text { sensor } \\
\text { ID }\end{array}$ & $\begin{array}{c}\text { transmit } \\
\text { power }\end{array}$ & $\begin{array}{c}\text { receiver } \\
\text { ID }\end{array}$ & $\begin{array}{c}\text { source } \\
\text { sensor } \\
\text { ID }\end{array}$ & $\begin{array}{c}\text { transmit } \\
\text { power }\end{array}$ & $\begin{array}{c}\text { receiver } \\
\text { ID }\end{array}$ & $\ldots$ & TB \\
\hline
\end{tabular}

Sensing data packet structure

\begin{tabular}{|c|c|c|c|c|}
\hline $\begin{array}{c}\text { source } \\
\text { sensor } \\
\text { ID }\end{array}$ & $\begin{array}{c}\text { destination } \\
\text { ID }\end{array}$ & reserve & data & TB \\
\hline
\end{tabular}

Figure 7 Data packet structures 
where DC is the duty cycle, $N_{\text {data }}$ the sampling data bits within one frame time, $N_{\text {oh }}$ the overhead bits within one frame time, $N_{\text {sync }}$ the synchronization bits within one frame time, $f_{\mathrm{c}}$ the communication data rate (bits per s) and $f_{\mathrm{s}}$ the sampling data rate (bits per s).

Generally the lower the duty cycle is, the better the MAC protocol is designed. The proposed protocol achieves a low duty cycle by reducing overhearing and idle listening.

\section{Performance evaluation}

The multiple capsule networks inside the small intestine were simulated using Qualnet 5.0. The energy consumption, latency, and duty cycle of the proposed MAC protocol were simulated and compared with B-MAC and star topology TDMA protocol. The results showed that the proposed MAC protocol outperforms B-MAC and the star topology TDMA in terms of energy consumption.

The small intestine is about $6 \mathrm{~m}$ in length. The mobility pattern is generated based on the small intestine model as shown in Figure 8. The moving speed of capsule devices is set to $0.2 \mathrm{~mm} / \mathrm{s}$. According to Chirwa et al. [14], this article estimates the path-loss using distance between any two capsule devices, $d$, according to Equation 12.

$$
\mathrm{PL}=d \times \frac{20 \mathrm{~dB}}{10 \mathrm{~cm}}
$$

$\mathrm{B}-\mathrm{MAC}$ is used in this article to compare with the proposed protocol. It is a contention-based MAC protocol employing an adaptive preamble sampling scheme to reduce duty cycle and minimize idle listening. A long preamble is used before data transmission. Sensor nodes follow independent sleeping schedule and periodically wake up to sense the channel. They will remain awake to receive the messages if they sense the activity on the channel or go to sleep if they do not detect activities. To get an optimal setting for B-MAC such that the smallest energy consumption can be achieved for the comparisons, the packet delivery ratio (PDR) vs. transmit power and the energy consumption vs. sleep interval for different number of nodes are simulated. From Figure 9 , it can be seen when the transmit power reaches $-5 \mathrm{dBm}$, the data recorder can receive $100 \%$ data. Therefore, $-5 \mathrm{dBm}$ is used as the B-MAC transmit power. The optimal B-MAC sleep interval, which achieves the smallest energy consumption for specific network size, is simulated in Figure 10.

Star topology TDMA is referred to Marinkovic et al.'s study [9]. The capsule devices and coordinator node communicate in a point-to-point mode. The frame is equally divided into small slots and each small slot is composed of the link packet, acknowledgement packet, and idle time. The small slots are equally assigned to capsule devices in advance. The transmit power of the star topology TDMA is set to $-2 \mathrm{dBm}$ which is the minimum transmit power required to ensure a delivery ratio of 1 .

For the proposed TDMA protocol simulation, 405 $\mathrm{MHz}$ is used as the wireless communication frequency. The payload size for synchronization, power detection, and scheduling assignment is defined as 10 bytes and the payload size for broadcasting and neighboring information uploading is defined as $10 \times N$, where $N$ is the number of the sensor devices. The total energy
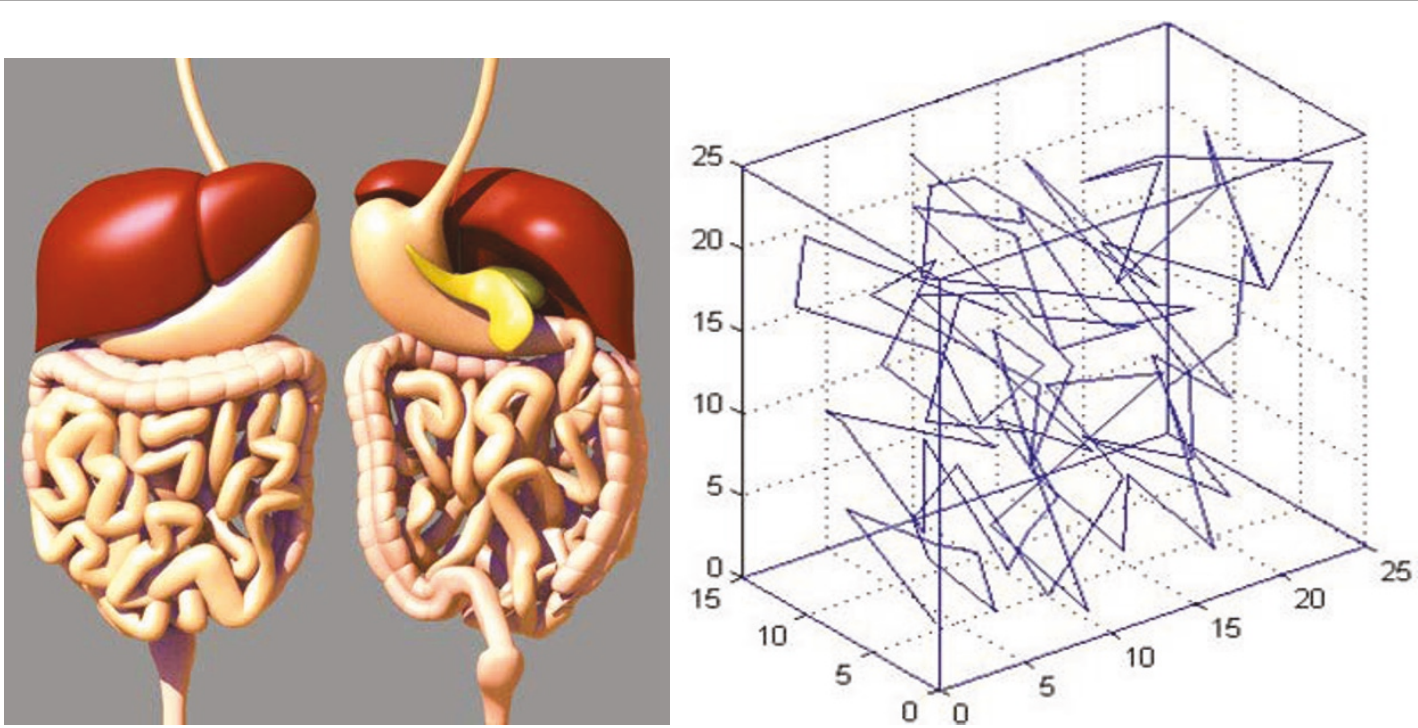

Figure 8 Mobility pattern for multiple capsule networks 


\section{Packet delivery ratio for B-MAC}

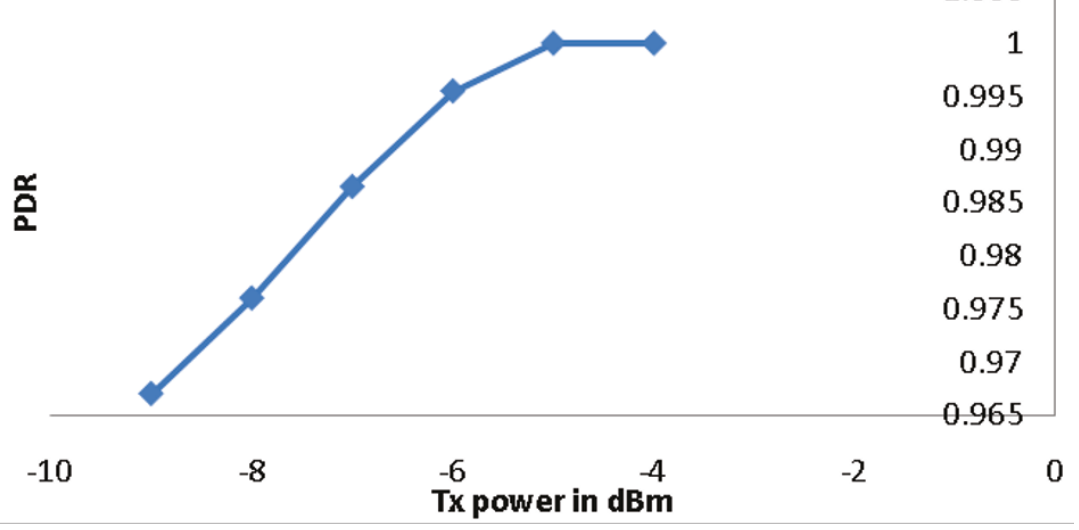

Figure 9 Packet delivery ratio vs. transmit power for B-MAC

consumption is calculated as the summation of the energy consumption of the transmit, receive, and idle states for all the capsule nodes. In our model, the power consumption in transmit mode is composed of the transmit circuitry power consumption and transmission signal power consumption, and the power consumption in receive/idle mode is equal to the receive circuitry power consumption. The simulation parameters used in Qualnet are listed in Table 2.

All the simulations for the three MAC protocols obtain the packet delivery ratio of 1 . Figure 11 shows the energy consumptions based on different number of capsules for the proposed TDMA, B-MAC, and the star topology TDMA. It can be seen that the energy consumption for the proposed TDMA is much smaller than that of B-MAC and star topology TDMA. The proposed TDMA scheme reduces the idle listening and overhearing compared with B-MAC. The adaptive power control enables the proposed protocol to use less transmit power for data communications.

Figure 12 shows the energy consumption vs. packet size from 0.5 to $3 \mathrm{kB}$. The same result that the proposed TDMA consumes much less energy than B-MAC and TDMA in [9] is obtained. Figure 13 gives the simulation of energy consumption vs. constant bit rate (CBR) packet interval. The proposed TDMA achieves significant performance gain for CBR packet interval from 200 $\mathrm{ms}$ to $1 \mathrm{~s}$.

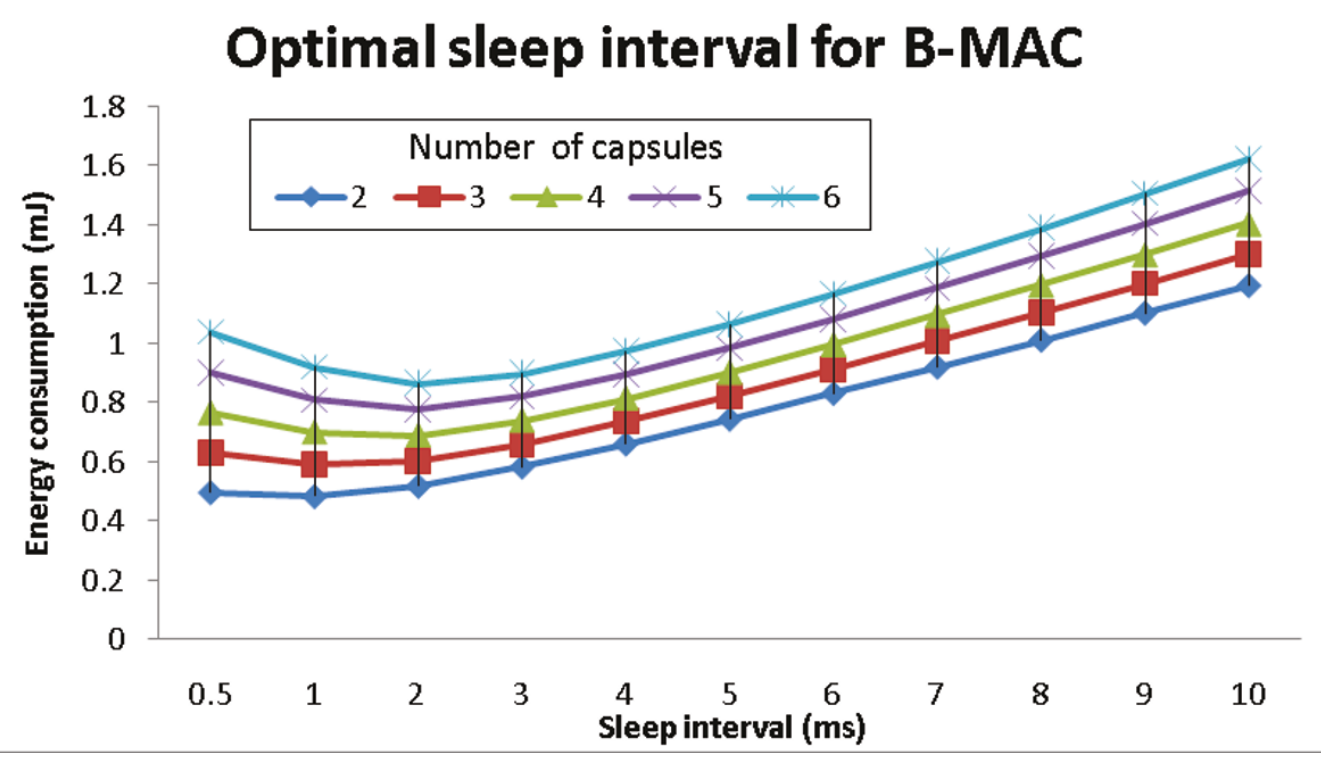

Figure 10 Optimal sleep interval for B-MAC for various network sizes 
Table 2 Parameters used in simulation.

\begin{tabular}{llll}
\hline Parameters & Value & Parameters & Value \\
\hline Area & $0.4 \mathrm{~m} \times 0.4 \mathrm{~m}$ & Transmit/receive circuitry power & $33 \mu \mathrm{W}$ \\
Number of capsules & $2 \mathrm{to} 6$ & MAC protocol & TDMA, B-MAC \\
Channel frequency & $405 \mathrm{MHz}$ & Mobility pattern & Small intestine \\
Pathloss model & Pathloss matrix & Items to send & 20000 \\
Radio type & ABSTRACT & Packet size & 512 Bytes to $3 \mathrm{kB}$ \\
Transmission power $(\mathrm{dBm})$ & Adaptive & CBR packet interval & 0.2 to $1 \mathrm{~s}$ \\
Energy model & GENERIC & Simulation time & $20000 \mathrm{~s}$ \\
Inefficiency factor $\alpha$ & 6.5 & &
\end{tabular}

\section{Energy consumption vs. number of capsules}

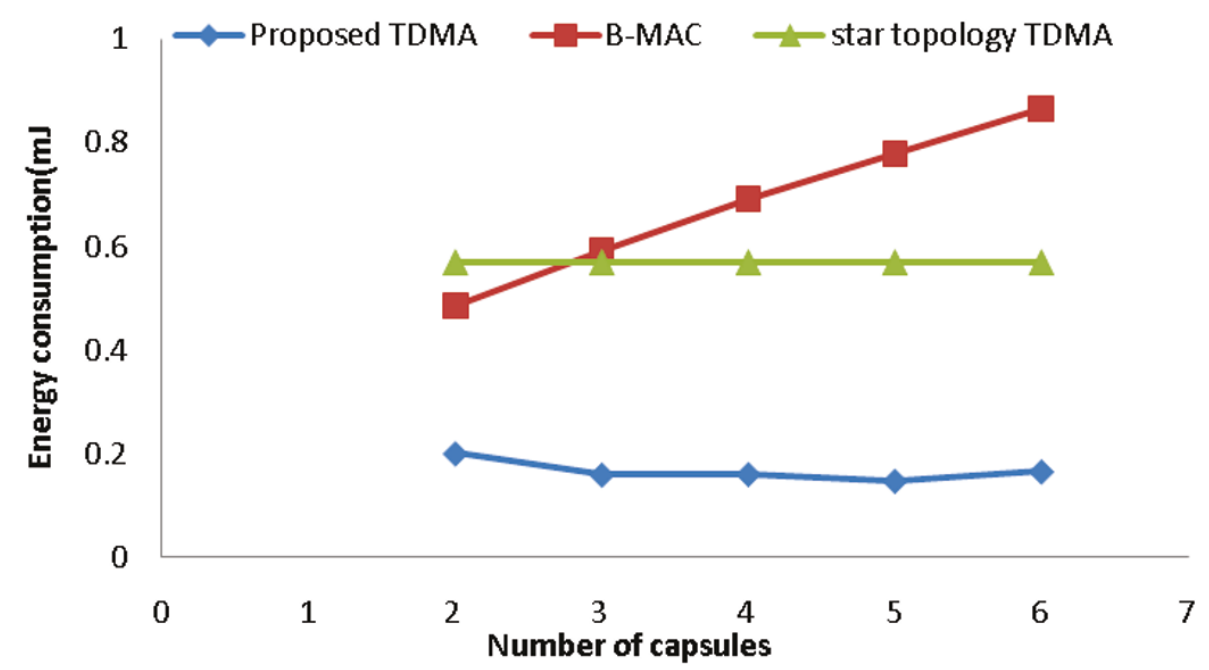

Figure 11 Energy consumption vs. number of capsules.

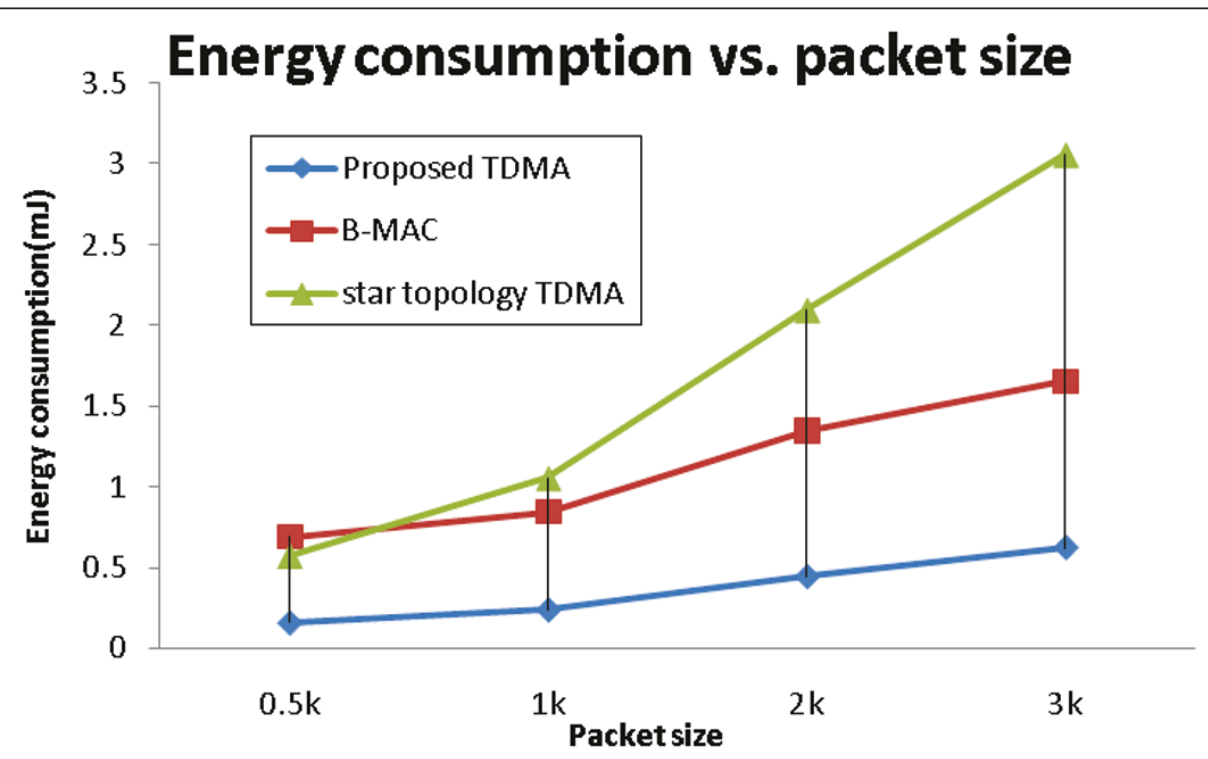

Figure 12 Energy consumption vs. packet size. 


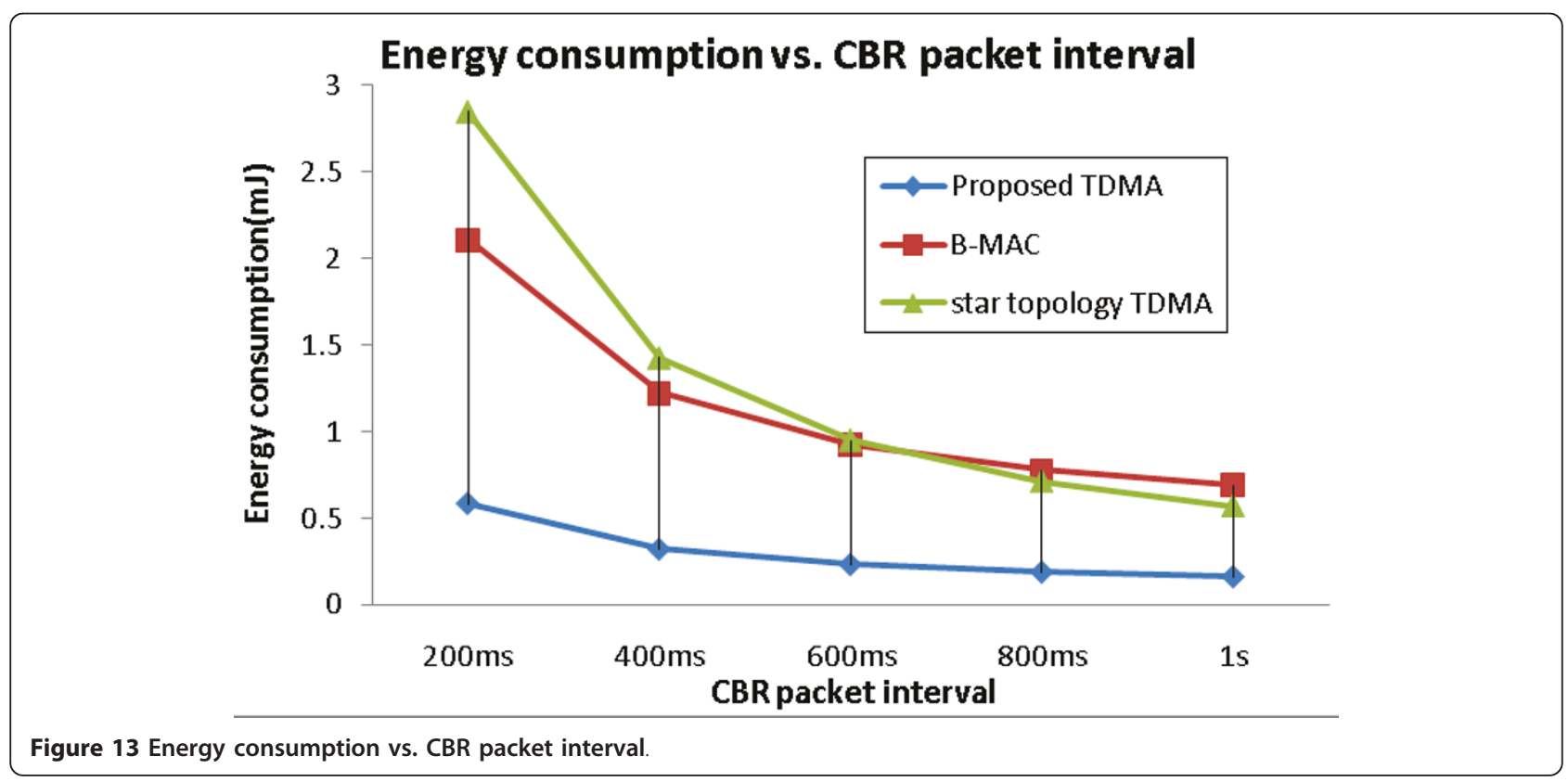

However, for the delay performance, the proposed TDMA shows worse performance than B-MAC and star topology TDMA. Figure 14 gives the average end-to-end delay. B-MAC and the star topology TDMA manage to consistently achieve a stable low latency for different network capacity. While for the proposed TDMA, the average end-to-end delay is very large and it increases when the network capacity becomes larger. This is due to the adaptive power control. The routing is calculated for achieving lower energy consumption. More hops can obtain lower energy consumption but cause larger delays at the same time. Another reason for the unfavorable delay performance is the TDMA frame format. In the TDMA frame format proposed, the data slots are followed by the control data slots. This would cause delay if the data is supposed to be sent during the control data section. The assigned slots would also introduce delay. For example, data transmission may be finished before the end of the slot, but other data transmission cannot start until the next slot. Further work will be done to minimize the latency of the proposed protocol.

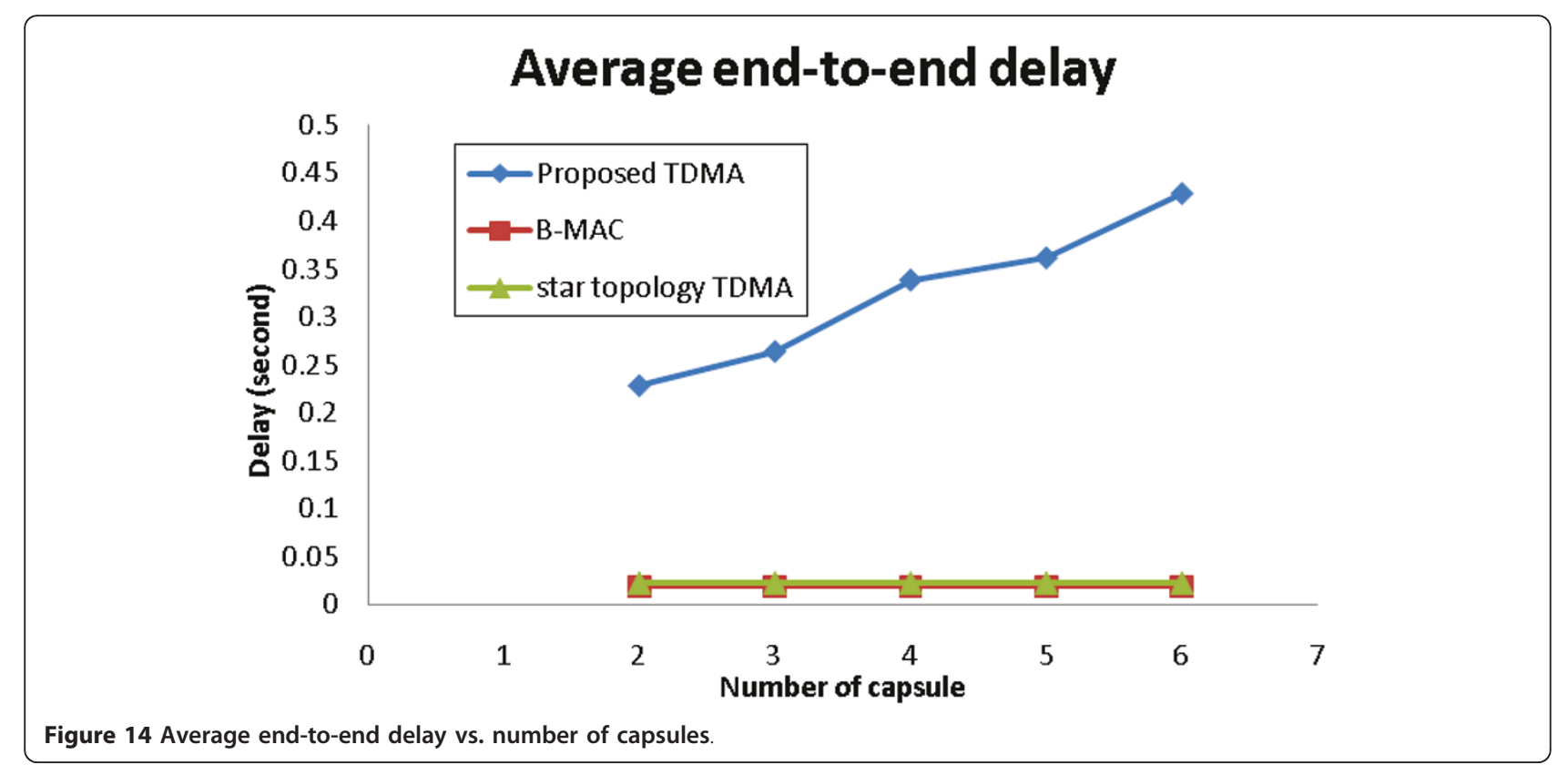




\section{Duty cycle comparison}

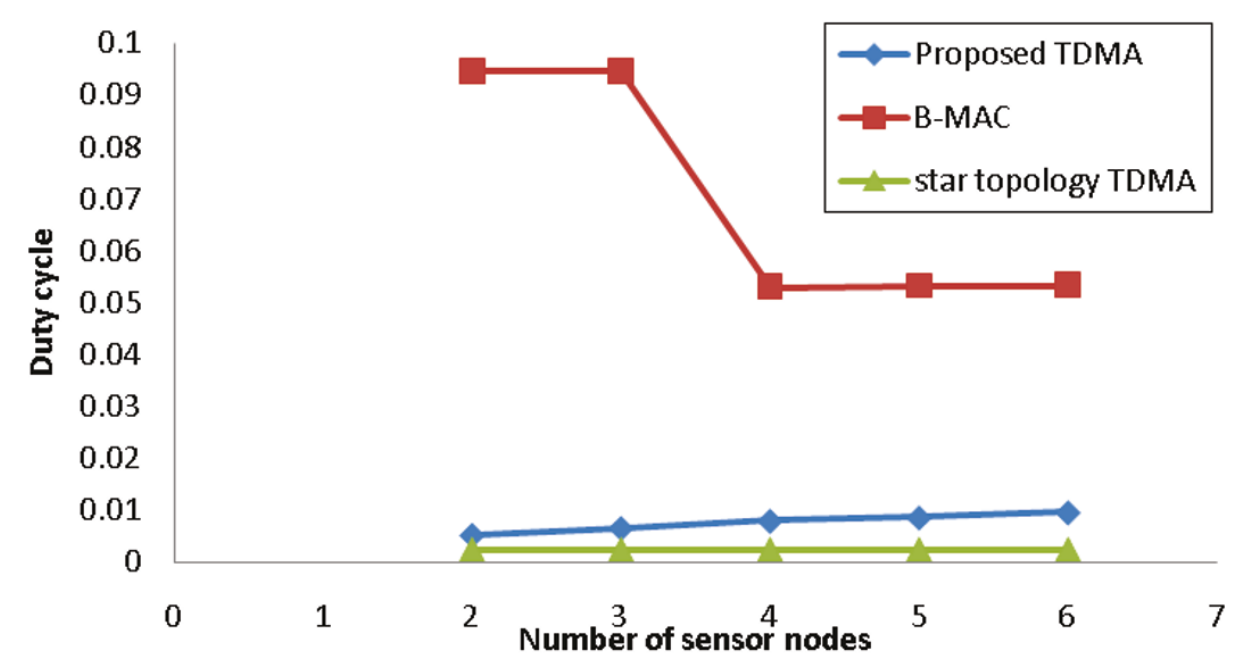

Figure 15 Duty cycle vs. number of nodes.

Figure 15 shows the duty cycle of the proposed TDMA, B-MAC, and star topology TDMA. The duty cycles of the two TDMA protocols are much lower than that of B-MAC, which means that the TDMA protocols sleep longer time than B-MAC. The duty cycle of the proposed TDMA is bigger than star topology TDMA because of larger overhead and the multiple transmissions of the same data. However, since the capsule utilizing the proposed protocol will be transmitting with lower transmission strength, the overall energy consumption will still be lower than when star topology TDMA is utilized as previously presented in Figure 11.

\section{Discussion and conclusion}

This article proposed a TDMA based MAC protocol for multiple capsule networks. A novel asymmetric up/ down link topology was used which is better than the pure star topology or distributed topology. In the proposed algorithm, capsules periodically send neighboring information to the coordinator node. The coordinator node then calculates the routing schedule for each capsule and sends these back to the capsules. The proposed protocol can avoid the idle listening and overhearing problems and many tasks are shifted to the coordinator node side to process. Simulation results showed that the proposed TDMA protocol achieves better performance than B-MAC and star topology TDMA in terms of energy consumption.

A few important issues pertaining to the operation of such in-body networks are discussed as follow. First, the objective is for health monitoring inside the human body, so safety and reliability are serious considerations. Safety and reliability must not be compromised even if higher cost is induced. Secondly, the frequency for power detection is dependent on the speed of the mobile sensors. If it is slow compared with the speed of capsules, then the path-loss may vary very much and the calculated transmit power becomes inaccurate. This inaccuracy will cause transmit power variations where; when it is set too small, it will cause packet loss; otherwise when it is set too high, it will result in energy wastage. Thirdly, the latency for the proposed TDMA is quite long. There are two reasons for this characteristic. One is the multi-hop communication and the other is the TDMA frame pattern. Further optimization for reducing the average end-to-end delay is expected and will be explored in future work. Finally, this article proposed a novel medium access solution for wireless capsule networks inside GI tract. This can also be a reference for the future complex body sensor networks. The future works would focus on optimizing power detection frequency and reducing the average end-toend delay.

\section{List of abbreviations}

BSN: body sensor networks; CBR: constant bit rate; ECG: electrocardiography; Gl: gastrointestinal; ID: identification; MAC: medium access control; PDR: packet delivery ratio; RSSI: received signal strength indication; TDMA: time division multiple access; WCE: wireless capsule endoscopy; WSN: wireless sensor networks.

\section{Acknowledgements}

This study has been supported by Singapore's Agency for Science, Technology and Research (ASTAR) under the grant No. 0821400036. 


\section{Author details}

${ }^{1}$ School of Mechanical and Aerospace Engineering, Nanyang Technological

University, Singapore ${ }^{2}$ School of Computer Engineering, Nanyang

Technological University, Singapore

\section{Competing interests}

The authors declare that they have no competing interests.

Received: 22 June 2011 Accepted: 4 August 2011

Published: 4 August 2011

\section{References}

1. Given Imaging (20 Jan 2011). Given Imaging http://www.givenimaging.com

2. L Lin, K-J Wong, S-L Tan, S-J Phee, Asymmetric multihop networks for multi-capsule communications within the gastrointestinal tract, in Wearable and Implantable Body Sensor Networks, 2009. BSN 2009. Sixth International Workshop on, pp. 82-86 (2009)

3. D Lan-Rong, W Yin-Yi, A wireless narrowband imaging chip for capsule endoscope. Biomed. Circuits Syst IEEE Trans. 4, 462-468 (2010)

4. K Kredo, P Mohapatra, Medium access control in wireless sensor networks. Comput Netw. 51, 961-994 (2007). doi:10.1016/j.comnet.2006.06.012

5. P Joseph, H Jason, C David, Versatile low power media access for wireless sensor networks. Presented at the Proceedings of the 2nd international conference on Embedded networked sensor systems, Baltimore, MD, USA (2004)

6. Y Wei, J Heidemann, D Estrin, An energy-efficient MAC protocol for wireless sensor networks, in INFOCOM 2002. Twenty-First Annual Joint Conference of the IEEE Computer and Communications Societies. Proceedings. IEEE. 3, 1567-1576 (2002)

7. WB Heinzelman, AP Chandrakasan, H Balakrishnan, An application-specific protocol architecture for wireless microsensor networks. Wireless Commun IEEE Trans. 1, 660-670 (2002). doi:10.1109/TWC.2002.804190

8. O Omeni, ACW Wong, AJ Burdett, C Toumazou, Energy efficient medium access protocol for wireless medical body area sensor networks. IEEE Trans Biomed Circuits Syst. 2, 251-259 (2008)

9. SJ Marinkovic, EM Popovici, C Spagnol, S Faul, WP Marnane, Energy-efficient low duty cycle mac protocol for wireless body area networks. IEEE Trans Infor Technol Biomed. 13, 915-925 (2009)

10. D Tijs van, L Koen, An adaptive energy-efficient MAC protocol for wireless sensor networks, Presented at the Proceedings of the 1st international conference on Embedded networked sensor systems, Los Angeles, California, USA, (2003)

11. V Rajendran, K Obraczka, J Garcia-Luna-Aceves, Energy-efficient, collisionfree medium access control for wireless sensor networks. Wireless Netw. 12, 63-78 (2006). doi:10.1007/s11276-006-6151-z

12. S Chatterjea, LFW van Hoesel, PJM Havinga, Al-LMAC an adaptive, information-centric and lightweight MAC protocol for wireless sensor networks, in Intelligent Sensors, Sensor Networks and Information Processing Conference, 2004. Proceedings of the 2004, pp. 381-388 (2004)

13. K HyungTae, L SuKyoung, Energy-efficient multi-hop transmission in body area networks, in Personal, Indoor and Mobile Radio Communications, 2009 IEEE 20th International Symposium on, pp. 2142-2146 (2009)

14. LC Chirwa, PA Hammond, S Roy, DRS Cumming, Electromagnetic radiation from ingested sources in the human intestine between $150 \mathrm{MHz}$ and 1.2 GHz. IEEE Trans Biomed Eng. 50, 484-92 (2003). doi:10.1109/ TBME.2003.809474

15. I McGregor, G Whyte, K Elgaid, Low complexity, 165 uW, 5 Mbit/s wideband radio front-end with range of several meters, in Microwave Symposium Digest, 2009. MTT '09. IEEE MTT-S International, pp. 397-400 (2009)

doi:10.1186/1687-1499-2011-54

Cite this article as: Lin et al.: Evaluation of a TDMA-based energy

efficient MAC protocol for multiple capsule networks. EURASIP Journal on

Wireless Communications and Networking 2011 2011:54

\section{Submit your manuscript to a SpringerOpen ${ }^{\mathcal{O}}$ journal and benefit from:}

- Convenient online submission

- Rigorous peer review

- Immediate publication on acceptance

- Open access: articles freely available online

- High visibility within the field

- Retaining the copyright to your article

Submit your next manuscript at $\gg$ springeropen.com 